\title{
Some new fixed point theorems in generalized intuitionistic fuzzy metric spaces
}

\author{
M. Rajeswari ${ }^{1}$, M. Jeyaraman ${ }^{2}$ and S. Durga ${ }^{3}$ \\ ${ }^{1}$ Mathematics Department, M.G.R College \\ 635130, Hosur, India \\ e-mail: rajimaths1980@gmail.com \\ ${ }^{2}$ Mathematics Department, Raja Doraisingam Govt. Arts College \\ 630561, Sivaganga, India \\ e-mail: jeya.math@gmail.com \\ ${ }^{2}$ Mathematics Department, Raja Doraisingam Govt. Arts College \\ 630561, Sivaganga, India \\ e-mail: durgasundaram96@gmail.com
}

This paper is dedicated to our advisors.

Received: 10 June 2019

Accepted: 10 October 2019

\begin{abstract}
The aim of this paper is to give some new fixed point theorems for contractive type mappings in generalized intuitionistic fuzzy metric spaces. The results presented improve some well known results in the literature.
\end{abstract}

Keywords: Complete, Compact, Fuzzy metric space, Intuitionistic fuzzy metric space.

2010 Mathematics Subject Classification: 54H25, 47H10, 47S40.

\section{Introduction}

The concept of fuzzy metric space was introduced initially by Kramosil and Michalek [6]. Later on, George and Veeramani [4] give the modified notion of fuzzy metric spaces due to Kramosil and Michalek and analyzed a Hausdorff topology of fuzzy metric spaces. In 2006, Sedghi and Shobe [8] defined $\mathcal{M}$-fuzzy metric spaces and proved a common fixed point theorem for weakly compatible mapping in this space. 
On the other hand, the concept of intuitionistic fuzzy set was introduced by Atanassov [1] as a generalization of fuzzy set. In 2004, Park [7] introduced the notion of intuitionistic fuzzy metric space. In this paper, we give some new fixed point theorems in generalized intuitionistic fuzzy metric spaces.

\section{Preliminaries}

Definition 2.1. The 5-tuble $(X, \mathcal{M}, \mathcal{N}, *, \diamond)$ is a generalized intuitionistic fuzzy metric space if $X$ is an arbitrary set, $*$ is a continuous $t$-norm, $\diamond$ is a continuous $t$-conorm and $\mathcal{M}, \mathcal{N}$ are fuzzy sets on $X^{3} \times[0,1)$ satisfying the following conditions: for all $x, y, z \in X$,

(i) $\mathcal{M}(x, y, z, t)+\mathcal{N}(x, y, z, t) \leq 1$,

(ii) $\mathcal{M}(x, y, z, 0)=0$,

(iii) $\mathcal{M}(x, y, z, t)=1$ for all $t>0$ iff $x=y=z$,

(iv) $\mathcal{M}(x, y, z, t)=\mathcal{M}(p\{x, y, z\}, t)$, when $p$ is the permutation function,

(v) $\mathcal{M}(x, y, z, t+s) \geq \mathcal{M}(x, y, a, t) * \mathcal{M}(a, z, z, s)$ for all $t, s>0$,

(vi) $\mathcal{M}(x, y, z,):.[0,1) \rightarrow[0,1]$ is left-continuous,

(vii) $\lim _{t \rightarrow \infty} \mathcal{M}(x, y, z, t)=1$,

(viii) $\mathcal{N}(x, y, z, 0)=1$,

(ix) $\mathcal{N}(x, y, z, t)=0$ for all $t>0$ iff $x=y=z$,

(x) $\mathcal{N}(x, y, z, t)=\mathcal{N}(p\{x, y, z\}, t)$, when $p$ is the permutation function,

(xi) $\mathcal{N}(x, y, z, t+s) \leq \mathcal{N}(x, y, a, t) \diamond \mathcal{N}(a, z, z, s)$ for all $t, s>0$,

(xii) $\mathcal{N}(x, y, z,):.[0,1) \rightarrow[0,1]$ is right-continuous,

(xiii) $\lim _{t \rightarrow \infty} \mathcal{N}(x, y, z, t)=0$.

Remark 2.2. By (iii) and (v), it is easy to show that $\mathcal{M}(x, y, z,$.$) is non-decreasing and by (ix)$ and (xi), it is easy to show that $\mathcal{N}(x, y, z,$.$) is non-increasing for all x, y, z \in X$.

Remark 2.3. Every generalized fuzzy metric space $(X, \mathcal{M}, *)$ is a generalized intuitionistic fuzzy metric space of the form $(X, \mathcal{M}, 1-\mathcal{M}, *, \diamond)$ such that $t$-norm $*$ and $t$-conorm $\diamond$ are associated, i.e. $x \diamond y=1-((1-x) *(1-y))$ for any $x, y \in[0,1]$.

Definition 2.4. A sequence $\left\{x_{n}\right\}$ in a generalized intuitionistic fuzzy metric space $(X, \mathcal{M}, \mathcal{N}, *, \diamond)$ is a Cauchy sequence iff

$$
\lim _{n \rightarrow \infty} \mathcal{M}\left(x_{n+p}, x_{n}, x_{n}, t\right)=1 \text { and } \lim _{n \rightarrow \infty} \mathcal{N}\left(x_{n+p}, x_{n}, x_{n}, t\right)=0
$$


for each $t>0$ and $p \in \mathbb{N}$. A sequence $\left\{x_{n}\right\}$ in $X$ is convergent to $x \in X$ if

$$
\lim _{n \rightarrow \infty} \mathcal{M}\left(x_{n}, x, x, t\right)=1 \text { and } \lim _{n \rightarrow \infty} \mathcal{N}\left(x_{n}, x, x, t\right)=0
$$

for each $t>0$. A generalized intuitionistic fuzzy metric space $(X, \mathcal{M}, \mathcal{N}, *, \diamond)$ is called complete, if every Cauchy sequence in $X$ is convergent. It is called compact, if every sequence in $X$ contains a convergent subsequence.

Lemma 2.5. Let $\lim _{n \rightarrow \infty} x_{n}=x, \lim _{n \rightarrow \infty} y_{n}=y$ and $\lim _{n \rightarrow \infty} z_{n}=z$. Then, for all $t>0$,

$$
\begin{array}{ll}
\lim _{n \rightarrow \infty} \inf \mathcal{M}\left(x_{n}, y_{n}, z_{n}, t\right) \geq \mathcal{M}(x, y, z, t), & \lim _{n \rightarrow \infty} \sup \mathcal{N}\left(x_{n}, y_{n}, z_{n}, t\right) \leq \mathcal{N}(x, y, z, t), \\
\lim _{n \rightarrow \infty} \sup \mathcal{M}\left(x_{n}, y_{n}, z_{n}, t\right) \leq \mathcal{M}(x, y, z, t), & \lim _{n \rightarrow \infty} \inf \mathcal{N}\left(x_{n}, y_{n}, z_{n}, t\right) \geq \mathcal{N}(x, y, z, t)
\end{array}
$$

Particularly, if $\mathcal{M}(x, y, z,$.$) and \mathcal{N}(x, y, z,$.$) are continuous at point t$, then

$$
\lim _{n \rightarrow \infty} \mathcal{M}\left(x_{n}, y_{n}, z_{n}, t\right)=\mathcal{M}(x, y, z, t) \text { and } \lim _{n \rightarrow \infty} \mathcal{N}\left(x_{n}, y_{n}, z_{n}, t\right)=\mathcal{N}(x, y, z, t)
$$

\section{Main results}

Definition 3.1. Let the function $\phi:[0, \infty) \rightarrow[0, \infty)$ satisfy the following conditions:

$\left(\phi_{1}\right): \phi(t)$ is strictly increasing, $\phi(0)=0$ and $\lim _{n \rightarrow \infty} \phi^{n}(t)=\infty$, for all $t>0$, where $\phi^{n}(t)$ denotes the $n$-th iterative function of $\phi(t)$. Then $\phi(t)>t$ and $\phi^{n}(t)>\phi^{n-1}(t)$ for $t>0$ and $n=1,2, \ldots$.

$\left(\phi_{2}\right): \lim _{t \rightarrow \infty}[\phi(t)-t]=\infty$.

Lemma 3.2. Let $*$ and $\diamond$ be continuous $t$-norm and t-conorm, respectively. Then, for each $\lambda \in(0,1)$, there is a sequence $\left\{\lambda_{n}\right\}$ in $(0,1)$ such that

$$
\left(1-\lambda_{n}\right) *\left(1-\lambda_{n}\right)>1-\lambda_{n-1} \text { and } \lambda_{n} \diamond \lambda_{n}<\lambda_{n-1}, n=1,2 \ldots,
$$

where $\lambda_{0}=\lambda$ (obviously, the sequence $\left\{\lambda_{n}\right\}$ satisfying the condition is decreasing).

Proof. Since $*$ is continuous at the point $(1,1)$ and $a * b \leq 1 * 1=1$, and since $\diamond$ is continuous at the point $(0,0)$ and $a \diamond b \geq 0 \diamond 0=0$ for all $a, b \in[0,1]$, we have

$$
\sup _{0<\mu<1}[(1-\mu) *(1-\mu)]=1 \quad \text { and } \quad \inf _{0<\mu<1} \mu \diamond \mu=0,
$$

respectively. Thus, for each $\lambda \in(0,1)$, there exists $\lambda_{1} \in(0,1)$ such that

$$
\left(1-\lambda_{1}\right) *\left(1-\lambda_{1}\right)>1-\lambda \text { and } \lambda_{1} \diamond \lambda_{1}<\lambda .
$$

Similarly, there exists $\lambda_{2} \in(0,1)$ such that $\left(1-\lambda_{2}\right) *\left(1-\lambda_{2}\right)>1-\lambda_{1}$ and $\lambda_{2} \diamond \lambda_{2}<\lambda_{1}$. Continuing this procedure, we can obtain a sequence $\left\{\lambda_{n}\right\} \subset(0,1)$ satisfying the condition. 
Lemma 3.3. Let $(X, \mathcal{M}, \mathcal{N}, *, \diamond)$ be a generalized intuitionistic fuzzy metric space. Let $T: X \rightarrow X$ be a mapping satisfying

$$
\mathcal{M}\left(T x, T y, T z, t_{1}\right)>\mathcal{M}\left(x, y, z, t_{1}\right) \quad \text { and } \quad \mathcal{N}\left(T x, T y, T z, t_{1}\right)<\mathcal{N}\left(x, y, z, t_{1}\right)
$$

where $t_{1}$ is a fixed positive number. Then, there exists a continuity point $t_{0}$ of $\mathcal{M}(x, y, z,$.$) and$ $\mathcal{N}(x, y, z,$.$) such that$

$$
\mathcal{M}\left(T x, T y, T z, t_{0}\right)>\mathcal{M}\left(x, y, z, t_{0}\right) \text { and } \mathcal{N}\left(T x, T y, T z, t_{0}\right)<\mathcal{N}\left(x, y, z, t_{0}\right) .
$$

Proof. Since $\mathcal{M}(T x, T y, T z,)-.\mathcal{M}(x, y, z,$.$) is left-continuous and \mathcal{N}(T x, T y, T z,$. $-\mathcal{N}(x, y, z,$.$) is right-continuous at point t_{1}$, there exists $t_{2}>0$ such that

$$
\mathcal{M}(T x, T y, T z, t)>\mathcal{M}(x, y, z, t) \text { and } \mathcal{N}(T x, T y, T z, t)<\mathcal{N}(x, y, z, t)
$$

for all $t \in\left[t_{2}, t_{1}\right]$. Note that the set of discontinuous points of $\mathcal{M}(x, y, z,$.$) and \mathcal{N}(x, y, z,$.$) is$ countable atmost. Hence, there exists $t_{0} \in\left[t_{2}, t_{1}\right]$ such that $\mathcal{M}(x, y, z,$.$) and \mathcal{N}(x, y, z,$.$) are$ continuous at $t_{0}$.

Theorem 3.4. Let $(X, \mathcal{M}, \mathcal{N}, *, \diamond)$ be a complete generalized intuitionistic fuzzy metric space. Let $T: X \rightarrow X$ be a mapping satisfying the following conditions:

(i) There exists $x_{0} \in X$ such that

$$
\lim _{t \rightarrow \infty} \mathcal{M}\left(x_{0}, T^{i} x_{0}, T^{i} x_{0}, t\right)=1, \lim _{t \rightarrow \infty} \mathcal{N}\left(x_{0}, T^{i} x_{0}, T^{i} x_{0}, t\right)=0, i=1,2, \ldots
$$

(ii) There exists a mapping $m: X \rightarrow \mathbb{N}$ such that for any $x, y, z \in X$

$$
\begin{aligned}
& \mathcal{M}\left(T^{m(x)} x, T^{m(x)} y, T^{m(x)} z, t\right) \geq \mathcal{M}(x, y, z, \phi(t)) \quad \text { and } \\
& \mathcal{N}\left(T^{m(x)} x, T^{m(x)} y, T^{m(x)} z, t\right) \leq \mathcal{N}(x, y, z, \phi(t))
\end{aligned}
$$

where the function $\phi(t)$ satisfies the conditions $\left(\phi_{1}\right)$ and $\left(\phi_{2}\right)$.

Then $T$ has a unique fixed point $x^{*}$ and the quasi-iterative sequence $\left\{x_{n}=T^{m\left(x_{n-1}\right)} x_{n-1}\right\}$ converges to $x^{*}$.

Proof. First, we prove that

$$
\sup _{s>0} \inf _{x \in O_{T}\left(x_{0}\right)} \mathcal{M}\left(x_{0}, x, x, s\right)=1 \quad \text { and } \inf _{s>0} \sup _{x \in O_{T}\left(x_{0}\right)} \mathcal{N}\left(x_{0}, x, x, s\right)=0
$$

where $O_{T}\left(x_{0}\right)=\left\{x_{0}, T x_{0}, T^{2} x_{0}, \ldots\right\}$ is called the orbit of $x_{0}$ for $T$. For any $n \in \mathbb{N}$ with $n>m\left(x_{0}\right)$, we can denote $n=k m\left(x_{0}\right)+s$, where $0 \leq s<m\left(x_{0}\right)$.

Note that $\phi(t)>t$ for all $t>0$ and $\lim _{t \rightarrow \infty}[\phi(t)-t]=1$.

By (3.4.1), we have

$$
\lim _{t \rightarrow \infty} \mathcal{M}\left(x_{0}, T^{i} x_{0}, T^{i} x_{0}, \phi(t)\right)=1 \quad \text { and } \quad \lim _{t \rightarrow \infty} \mathcal{N}\left(x_{0}, T^{i} x_{0}, T^{i} x_{0}, \phi(t)\right)=0
$$

for $i=1,2, \ldots, m\left(x_{0}\right)$ and

$$
\lim _{t \rightarrow \infty} \mathcal{M}\left(x_{0}, T^{i} x_{0}, T^{i} x_{0}, \phi(t)-t\right)=1 \quad \text { and } \quad \lim _{t \rightarrow \infty} \mathcal{N}\left(x_{0}, T^{i} x_{0}, T^{i} x_{0}, \phi(t)-t\right)=0
$$


Moreover, by Lemma 3.2, for any $\lambda \in(0,1)$, there is a sequence $\left\{\lambda_{n}\right\}$ in $(0,1)$ such that $\left(1-\lambda_{n}\right) *\left(1-\lambda_{n}\right)>1-\lambda_{n-1}$ and $\lambda_{n} \diamond \lambda_{n}<\lambda_{n-1}, n=1,2 \ldots$

Thus, it follows from (3.4.4) and (3.4.5) that for a given $\lambda_{k}$, there exists $t_{0}>0$ such that

$$
\begin{gathered}
\min _{1 \leq i \leq m\left(x_{0}\right)} \mathcal{M}\left(x_{0}, T^{i} x_{0}, T^{i} x_{0}, \phi(t)\right)>1-\lambda_{k} \quad \text { and } \\
\max _{1 \leq i \leq m\left(x_{0}\right)} \mathcal{N}\left(x_{0}, T^{i} x_{0}, T^{i} x_{0}, \phi(t)\right)<\lambda_{k}, \\
\mathcal{M}\left(x_{0}, T^{m\left(x_{0}\right)} x_{0}, T^{m\left(x_{0}\right)} x_{0}, \phi(t)-t\right)>1-\lambda_{k} \quad \text { and } \\
\mathcal{N}\left(x_{0}, T^{m\left(x_{0}\right)} x_{0}, T^{m\left(x_{0}\right)} x_{0}, \phi(t)-t\right)<\lambda_{k}
\end{gathered}
$$

for all $t>t_{0}$. So, by (3.4.2), for all $t>t_{0}$, we have

$$
\begin{aligned}
& \mathcal{M}\left(x_{0}, T^{n} x_{0}, T^{n} x_{0}, \phi(t)\right)=\mathcal{M}\left(x_{0}, T^{k m\left(x_{0}\right)+s} x_{0}, T^{k m\left(x_{0}\right)+s} x_{0}, \phi(t)\right) \\
& \geq \mathcal{M}\left(x_{0}, T^{m\left(x_{0}\right)} x_{0}, T^{m\left(x_{0}\right)} x_{0}, \phi(t)-t\right) * \mathcal{M}\left(T^{m\left(x_{0}\right)} x_{0}, T^{k m\left(x_{0}\right)+s} x_{0}, T^{k m\left(x_{0}\right)+s} x_{0}, t\right) \\
& \geq \mathcal{M}\left(x_{0}, T^{m\left(x_{0}\right)} x_{0}, T^{m\left(x_{0}\right)} x_{0}, \phi(t)-t\right) * \mathcal{M}\left(x_{0}, T^{(k-1) m\left(x_{0}\right)+s} x_{0}, T^{(k-1) m\left(x_{0}\right)+s} x_{0}, \phi(t)\right) \\
& \geq \cdots \geq \mathcal{M}\left(x_{0}, T^{m\left(x_{0}\right)} x_{0}, T^{m\left(x_{0}\right)} x_{0}, \phi(t)-t\right) * \ldots{ }^{(k)} * \mathcal{M}\left(x_{0}, T^{m\left(x_{0}\right)} x_{0}, T^{m\left(x_{0}\right)} x_{0}, \phi(t)-t\right) \\
& * \mathcal{M}\left(x_{0}, T^{s} x_{0}, T^{s} x_{0}, \phi(t)\right) \\
& >\left(1-\lambda_{k}\right) * \ldots^{(k+1) \cdot} *\left(1-\lambda_{k}\right) \\
& >\left(1-\lambda_{k-1}\right) * \ldots{ }^{(k)} \cdot *\left(1-\lambda_{k-1}\right) \\
& >\ldots>\left(1-\lambda_{1}\right) *\left(1-\lambda_{1}\right)>1-\lambda . \\
& \mathcal{N}\left(x_{0}, T^{n} x_{0}, T^{n} x_{0}, \phi(t)\right)=\mathcal{N}\left(x_{0}, T^{k m\left(x_{0}\right)+s} x_{0}, T^{k m\left(x_{0}\right)+s} x_{0}, \phi(t)\right) \\
& \leq \mathcal{N}\left(x_{0}, T^{m\left(x_{0}\right)} x_{0}, T^{m\left(x_{0}\right)} x_{0}, \phi(t)-t\right) \diamond \mathcal{N}\left(T^{m\left(x_{0}\right)} x_{0}, T^{k m\left(x_{0}\right)+s} x_{0}, T^{k m\left(x_{0}\right)+s} x 0, t\right) \\
& \leq \mathcal{N}\left(x_{0}, T^{m\left(x_{0}\right)} x_{0}, T^{m\left(x_{0}\right)} x_{0}, \phi(t)-t\right) \diamond \mathcal{N}\left(x_{0}, T^{(k-1) m\left(x_{0}\right)+s} x_{0}, T^{(k-1) m\left(x_{0}\right)+s} x_{0}, \phi(t)\right) \\
& \leq \ldots \leq \mathcal{N}\left(x_{0}, T^{m\left(x_{0}\right)} x_{0}, T^{m\left(x_{0}\right)} x_{0}, \phi(t)-t\right) \diamond \ldots{ }^{(k)} \diamond \mathcal{N}\left(x_{0}, T^{m\left(x_{0}\right)} x_{0}, T^{m\left(x_{0}\right)} x_{0}, \phi(t)-t\right) \\
& \diamond \mathcal{N}\left(x_{0}, T^{s} x_{0}, T^{s} x_{0}, \phi(t)\right) \\
& <\lambda_{k} \diamond \ldots{ }^{(k+1)} \diamond \lambda_{k} \\
& <\lambda_{k-1} \diamond \ldots{ }^{(k)} \diamond \lambda_{k-1} \\
& <\ldots<\lambda_{1} \diamond \lambda_{1}<\lambda \text {. }
\end{aligned}
$$

Therefore, for all $t>t_{0}$,

$$
\inf _{x \in O_{T}\left(x_{0}\right)} \mathcal{M}\left(x_{0}, x, x, \phi(t)\right) \geq 1-\lambda \text { and } \sup _{x \in O_{T}\left(x_{0}\right)} \mathcal{N}\left(x_{0}, x, x, \phi(t)\right) \leq \lambda
$$

and hence

$$
\sup _{s>0} \inf _{x \in O_{T}\left(x_{0}\right)} \mathcal{M}\left(x_{0}, x, x, s\right)=1-\lambda \text { and } \inf _{s>0} \sup _{x \in O_{T}\left(x_{0}\right)} \mathcal{N}\left(x_{0}, x, x, s\right)=\lambda .
$$

By the arbitrariness of $\lambda$, we have

$$
\sup _{s>0} \inf _{x \in O_{T}\left(x_{0}\right)} \mathcal{M}\left(x_{0}, x, x, s\right)=1 \text { and } \inf _{s>0} \sup _{x \in O_{T}\left(x_{0}\right)} \mathcal{N}\left(x_{0}, x, x, s\right)=0 .
$$


Next, we prove that the quasi-iterative sequence $\left\{x_{n}=T^{m\left(x_{n-1}\right)} x_{n-1}\right\}_{n=1}^{\infty}$ is a Cauchy sequence.

For convenience, put $m_{i}=m\left(x_{i}\right), i=0,1,2 \ldots$ Then, by (3.4.1), for all $t>0$, we have

$$
\begin{aligned}
& \mathcal{M}\left(x_{n}, x_{n+p}, x_{n+p}, t\right)=\mathcal{M}\left(T^{m_{n-1}} x_{n-1}, T^{m_{n+p-1}+m_{n+p-2} \cdots+m_{n-1}} x_{n-1},\right. \\
& \left.\quad T^{m_{n+p-1}+m_{n+p-2}+\cdots+m_{n-1}} x_{n-1}, t\right) \\
& \geq \mathcal{M}\left(x_{n-1}, T^{m_{n+p-1}+\cdots+m_{n}} x_{n-1}, T^{m_{n+p-1}+\cdots+m_{n}} x_{n-1}, \phi(t)\right) \\
& \geq \mathcal{M}\left(x_{n-2}, T^{m_{n+p-1}+\cdots+m_{n}} x_{n-2}, T^{m_{n+p-1}+\cdots+m_{n}} x_{n-2}, \phi^{2}(t)\right) \\
& \geq \cdots \geq \mathcal{M}\left(x_{0}, T^{m_{n+p-1}+\cdots+m_{n}} x_{0}, T^{m_{n+p-1}+\cdots+m_{n}} x_{0}, \phi^{n}(t)\right) \\
& \geq \inf _{x \in O_{T}\left(x_{0}\right)} \mathcal{M}\left(x_{0}, x, x, \phi^{n}(t)\right) \\
& \geq \sup _{0<s<\phi^{n}(t)} \inf _{x \in O_{T}\left(x_{0}\right)} \mathcal{M}\left(x_{0}, x, x, s\right) \\
& \mathcal{N}\left(x_{n}, x_{n+p}, x_{n+p}, t\right)=\mathcal{N}\left(T^{m_{n-1}} x_{n-1}, T^{m_{n+p-1}+m_{n+p-2} \cdots+m_{n-1}} x_{n-1},\right. \\
& \left.\quad T^{m_{n+p-1}+m_{n+p-2}+\cdots+m_{n-1}} x_{n-1}, t\right) \\
& \leq \mathcal{N}\left(x_{n-1}, T^{m_{n+p-1}+\cdots+m_{n}} x_{n-1}, T^{m_{n+p-1}+\cdots+m_{n}} x_{n-1}, \phi(t)\right) \\
& \leq \mathcal{N}\left(x_{n-2}, T^{m_{n+p-1}+\cdots+m_{n}} x_{n-2}, T^{m_{n+p-1}+\cdots+m_{n}} x_{n-2}, \phi^{2}(t)\right) \\
& \leq \cdots \leq \mathcal{N}\left(x_{0}, T^{m_{n+p-1}+\cdots+m_{n}} x_{0}, T^{m_{n+p-1}+\cdots+m_{n}} x_{0}, \phi^{n}(t)\right) \\
& \leq \sup _{x \in O_{T}\left(x_{0}\right)} \mathcal{N}\left(x_{0}, x, x, \phi^{n}(t)\right) \\
& \leq \inf _{0<s<\phi^{n}(t)} \sup _{x \in O_{T}\left(x_{0}\right)} \mathcal{N}\left(x_{0}, x, x, s\right)
\end{aligned}
$$

Then, by condition $\left(\phi_{1}\right)$ and (3.4.3)

$$
\lim _{n \rightarrow \infty} \mathcal{M}\left(x_{n}, x_{n+p}, x_{n+p}, t\right)=1 \text { and } \lim _{n \rightarrow \infty} \mathcal{N}\left(x_{n}, x_{n+p}, x_{n+p}, t\right)=0
$$

for all $t>0$. This means that $\left\{x_{n}\right\}$ is a Cauchy sequence in $X$. By the completeness of $X$, there exists $\lim _{n \rightarrow \infty} x_{n}=x^{*} \in X$. Now, we prove that $x^{*}$ is the unique fixed point $T^{m^{*}}$, where $m^{*}=m\left(x^{*}\right)$. By (v) and (xi) in Definition 2.1, and (3.4.2), we have

$$
\begin{aligned}
\mathcal{M}\left(x^{*}, T^{m^{*}} x^{*}, T^{m^{*}} x^{*}, t\right) & \geq \mathcal{M}\left(x^{*}, T^{m^{*}} x_{n}, T^{m^{*}} x_{n}, t / 2\right) * \mathcal{M}\left(T^{m^{*}} x_{n}, T^{m^{*}} x^{*}, T^{m^{*}} x^{*}, t / 2\right) \\
& \geq \mathcal{M}\left(x^{*}, T^{m^{*}} x_{n}, T^{m^{*}} x_{n}, t / 2\right) * \mathcal{M}\left(x_{n}, T^{m^{*}} x^{*}, T^{m^{*}} x^{*}, \phi(t / 2)\right) \\
\mathcal{N}\left(x^{*}, T^{m^{*}} x^{*}, T^{m^{*}} x^{*}, t\right) & \leq \mathcal{N}\left(x^{*}, T^{m^{*}} x_{n}, T^{m^{*}} x_{n}, t / 2\right) \diamond \mathcal{N}\left(T^{m^{*}} x_{n}, T^{m^{*}} x^{*}, T^{m^{*}} x^{*}, t / 2\right) \\
& \leq \mathcal{N}\left(x^{*}, T^{m^{*}} x_{n}, T^{m^{*}} x_{n}, t / 2\right) \diamond \mathcal{N}\left(x_{n}, T^{m^{*}} x^{*}, T^{m^{*}} x^{*}, \phi(t / 2)\right)
\end{aligned}
$$

It is easy to prove that

$$
\lim _{n \rightarrow \infty} \mathcal{M}\left(x^{*}, T^{m^{*}} x_{n}, T^{m^{*}} x_{n}, u\right)=1 \text { and } \lim _{n \rightarrow \infty} \mathcal{N}\left(x^{*}, T^{m^{*}} x_{n}, T^{m^{*}} x_{n}, u\right)=0
$$

for all $u>0$. In fact,

$$
\mathcal{M}\left(x^{*}, T^{m^{*}} x_{n}, T^{m^{*}} x_{n}, u\right) \geq \mathcal{M}\left(x^{*}, x_{n}, x_{n}, u / 2\right) * \mathcal{M}\left(x^{*}, T^{m^{*}} x_{n}, T^{m^{*}} x_{n}, u / 2\right)
$$




$$
\begin{aligned}
& \geq \mathcal{M}\left(x^{*}, x_{n}, x_{n}, u / 2\right) * \mathcal{M}\left(T^{m_{n-1}} x_{n-1}, T^{m_{n-1}+m^{*}} x_{n-1}, T^{m_{n-1}+m^{*}} x_{n}, u / 2\right) \\
& \geq \mathcal{M}\left(x^{*}, x_{n}, x_{n}, u / 2\right) * \mathcal{M}\left(x_{n-1}, T^{m^{*}} x_{n-1}, T^{m^{*}} x_{n-1}, \phi(u / 2)\right) \\
& \geq \cdots \geq \mathcal{M}\left(x^{*}, x_{n}, x_{n}, u / 2\right) * \mathcal{M}\left(x_{0}, T^{m^{*}} x_{0}, T^{m^{*}} x_{0}, \phi^{n}(u / 2)\right) \rightarrow 1 \\
& \mathcal{N}\left(x^{*}, T^{m^{*}} x_{n}, T^{m^{*}} x_{n}, u\right) \leq \mathcal{N}\left(x^{*}, x_{n}, x_{n}, u / 2\right) \diamond \mathcal{N}\left(x^{*}, T^{m^{*}} x_{n}, T^{m^{*}} x_{n}, u / 2\right) \\
& \leq \mathcal{N}\left(x^{*}, x_{n}, x_{n}, u / 2\right) \diamond \mathcal{N}\left(T^{m_{n-1}} x_{n-1}, T^{m_{n-1}+m^{*}} x_{n-1}, T^{m_{n-1}+m^{*}} x_{n}, u / 2\right) \\
& \leq \mathcal{N}\left(x^{*}, x_{n}, x_{n}, u / 2\right) \diamond \mathcal{N}\left(x_{n-1}, T^{m^{*}} x_{n-1}, T^{m^{*}} x_{n-1}, \phi(u / 2)\right) \\
& \leq \cdots \leq \mathcal{N}\left(x^{*}, x_{n}, x_{n}, u / 2\right) \diamond \mathcal{N}\left(x_{0}, T^{m^{*}} x_{0}, T^{m^{*}} x_{0}, \phi^{n}(u / 2)\right) \rightarrow 0
\end{aligned}
$$

Thus, letting $n \rightarrow \infty$ on the right-hand sides of (3.4.6) and (3.4.7), and noting the continuity of $*$ and $\diamond$, we have

$$
\mathcal{M}\left(x^{*}, T^{m^{*}} x^{*}, T^{m^{*}} x^{*}, t\right)=1 \quad \text { and } \quad \mathcal{N}\left(x^{*}, T^{m^{*}} x^{*} T^{m^{*}} x^{*}, t\right)=0
$$

for all $t>0$.

This implies that $T^{m^{*}} x^{*}=x^{*}$, i.e., $x^{*}$ is a fixed point $T^{m\left(x^{*}\right)}$.

To show uniqueness, assume that $T^{m\left(x^{*}\right)}(y)=y, T^{m\left(x^{*}\right)}(z)=z$ for some $y, z \in X$.

Then,

$$
\begin{gathered}
\mathcal{M}\left(x^{*}, y, z, t\right)=\mathcal{M}\left(T^{m\left(x^{*}\right)}\left(x^{*}\right), T^{m\left(x^{*}\right)}(y), T^{m\left(x^{*}\right)}(z), t\right) \geq \mathcal{M}\left(x^{*}, y, z, \phi(t)\right), \\
\mathcal{N}\left(x^{*}, y, z, t\right)=\mathcal{N}\left(T^{m\left(x^{*}\right)}\left(x^{*}\right), T^{m\left(x^{*}\right)}(y), T^{m\left(x^{*}\right)}(z), t\right) \leq \mathcal{N}\left(x^{*}, y, z, \phi(t)\right) .
\end{gathered}
$$

On the other hand, as $\mathcal{M}\left(x^{*}, y, z, t\right)$ is non-decreasing and $\mathcal{N}\left(x^{*}, y, z, t\right)$ is non-increasing, we have

$$
\mathcal{M}\left(x^{*}, y, z, t\right) \leq \mathcal{M}\left(x^{*}, y, z, \phi(t)\right) \quad \text { and } \quad \mathcal{N}\left(x^{*}, y, z, t\right) \geq \mathcal{N}\left(x^{*}, y, z, \phi(t)\right),
$$

respectively.

Thus,

$$
\begin{aligned}
\mathcal{M}\left(x^{*}, y, z, t\right) & =\mathcal{M}\left(x^{*}, y, z, \phi(t)\right)=\mathcal{M}\left(x^{*}, y, z, \phi^{n}(t)\right), \\
\mathcal{N}\left(x^{*}, y, z, t\right) & =\mathcal{N}\left(x^{*}, y, z, \phi(t)\right)=\mathcal{N}\left(x^{*}, y, z, \phi^{n}(t)\right)
\end{aligned}
$$

for all $t>0$.

Hence, by condition $\left(\phi_{1}\right)$, and (vii) and (xiii) in Definition 2.1, we have

$$
\mathcal{M}\left(x^{*}, y, z, t\right)=1 \quad \text { and } \quad \mathcal{N}\left(x^{*}, y, z, t\right)=0,
$$

i.e. $x^{*}=y=z$. Finally, we prove $x^{*}$ is the unique fixed point of $T$,

Since $T^{m\left(x^{*}\right)} x^{*}=x^{*}$, it follows that $T x^{*}=T\left(T^{m^{*}} x^{*}\right)=T^{m^{*}}\left(T x^{*}\right)$.

Hence, $T x^{*}=x^{*}$.

The uniqueness is obvious. This completes the proof.

From Theorem 3.4, we can immediately obtain the following consequence. 
Corollary 3.5. Let $(X, \mathcal{M}, \mathcal{N}, *, \diamond)$ be a complete generalized intuitionistic fuzzy metric space. Let $T: X \rightarrow X$ be a mapping satisfying the following conditions:

(i) There exists $x_{0} \in X$ such that

$$
\lim _{t \rightarrow \infty} \mathcal{M}\left(x_{0}, T^{i} x_{0}, T^{i} x_{0}, t\right)=1
$$

and

$$
\lim _{t \rightarrow \infty} \mathcal{N}\left(x_{0}, T^{i} x_{0}, T^{i} x_{0}, t\right)=0, i=1,2, \ldots
$$

(ii) There exists a mapping $m: X \rightarrow \mathcal{N}$ such that for any $x, y, z \in X$

$$
\begin{gathered}
\mathcal{M}\left(T^{m(x)} x, T^{m(x)} y, T^{m(x)} z, t\right) \geq \mathcal{M}(x, y, z, t / k), \\
\mathcal{N}\left(T^{m(x)} x, T^{m(x)} y, T^{m(x)} z, t\right) \leq \mathcal{N}(x, y, z, t / k)
\end{gathered}
$$

where $0<k<1$.

Then the conclusion of Theorem 3.4 remains true.

Proof. Taking $\phi(t)=t / k$. Obviously, $\phi(t)$ satisfies the conditions $\left(\phi_{1}\right)$ and $\left(\phi_{2}\right)$. Therefore, the conclusion directly follows from Theorem 3.4.

Corollary 3.6. Let $(X, \mathcal{M}, \mathcal{N}, *, \diamond)$ be a complete generalized intuitionistic fuzzy metric space. Let $T: X \rightarrow X$ be a mapping. If there exists a mapping $m: X \rightarrow \mathbb{N}$ such that for any $x, y, z \in X$,

$$
\begin{gathered}
\mathcal{M}\left(T^{m(x)} x, T^{m(x)} y, T^{m(x)} z, t\right) \geq \mathcal{M}(x, y, z, \phi(t)), \\
\mathcal{N}\left(T^{m(x)} x, T^{m(x)} y, T^{m(x)} z, t\right) \leq \mathcal{N}(x, y, z, \phi(t)),
\end{gathered}
$$

where the function $\phi(t)$ satisfies the conditions $\left(\phi_{1}\right)$ and $\left(\phi_{2}\right)$. Then $T$ has a unique fixed point $x^{*}$ and the iterative sequence $\left\{T^{n} x\right\}$ converges to $x^{*}$ for every $x \in X$.

Proof. By Theorem 3.4, we only need to show that the iterative sequence $\left\{T^{n} x\right\}$ converges to $x^{*}$. For $n \in \mathbb{N}$ with $n>m\left(x^{*}\right), n=k m\left(x^{*}\right)+s, 0 \leq s<m\left(x^{*}\right)$.

Since

$$
\begin{aligned}
\mathcal{M}\left(x^{*}, T^{n} x, T^{n} x, t\right) & =\mathcal{M}\left(T^{m\left(x^{*}\right)} x^{*}, T^{k m\left(x^{*}\right)+s} x, T^{k m\left(x^{*}\right)+s} x, t\right) \\
& \geq \mathcal{M}\left(x^{*}, T^{(k-1) m\left(x^{*}\right)+s} x, T^{(k-1) m\left(x^{*}\right)+s} x, \phi(t)\right) \\
& \geq \cdots \geq \mathcal{M}\left(x^{*}, T^{s} x, T^{s} x, \phi^{n}(t)\right) \rightarrow 1, \\
\mathcal{N}\left(x^{*}, T^{n} x, T^{n} x, t\right) & =\mathcal{N}\left(T^{m\left(x^{*}\right)} x^{*}, T^{k m\left(x^{*}\right)+s} x, T^{k m\left(x^{*}\right)+s} x, t\right) \\
& \leq \mathcal{N}\left(x^{*}, T^{(k-1) m\left(x^{*}\right)+s} x, T^{(k-1) m\left(x^{*}\right)+s} x, \phi(t)\right) \\
& \leq \cdots \leq \mathcal{N}\left(x^{*}, T^{s} x, T^{s} x, \phi^{n}(t)\right) \rightarrow 0,
\end{aligned}
$$

so, $\lim _{n \rightarrow \infty} \mathcal{M}\left(x^{*}, T^{n} x, T^{n} x, t\right)=1$ and $\lim _{n \rightarrow \infty} \mathcal{N}\left(x^{*}, T^{n} x, T^{n} x, t\right)=0$ for all $t>0$, i.e., $T^{n} x \rightarrow x^{*}$. 
Theorem 3.7. Let $(X, \mathcal{M}, \mathcal{N}, *, \diamond)$ be a compact generalized intuitionistic fuzzy metric space. Let $T: X \rightarrow X$ be a continuous mapping satisfying

$$
\mathcal{M}(T x, T y, T z, .)>\min \{\mathcal{M}(x, T x, T x, .), \mathcal{M}(y, T y, T y, .), \mathcal{M}(z, T z, T z, .), \mathcal{M}(x, y, z, .)\}
$$

$$
\mathcal{N}(T x, T y, T z, .)<\max \{\mathcal{N}(x, T x, T x, .), \mathcal{N}(y, T y, T y, .), \mathcal{N}(z, T z, T z, .), \mathcal{N}(x, y, z, .)\}
$$

for all $x \neq y \neq z$. If there exists $x_{0} \in X$ such that $\left\{T^{n} x_{0}\right\}_{n=1}^{\infty}$ has an accumulation point $x^{*} \in X$ and for all $t>0, n=1,2, \ldots$.

$$
\begin{aligned}
& \mathcal{M}\left(T^{n-1} x_{0}, T^{n} x_{0}, T^{n} x_{0}, t\right) \leq \mathcal{M}\left(T^{n} x_{0}, T^{n+1} x_{0}, T^{n+1} x_{0}, t\right), \\
& \mathcal{N}\left(T^{n-1} x_{0}, T^{n} x_{0}, T^{n} x_{0}, t\right) \geq \mathcal{N}\left(T^{n} x_{0}, T^{n+1} x_{0}, T^{n+1} x_{0}, t\right)
\end{aligned}
$$

then $x^{*}$ is the unique fixed point of $T$ and $\lim _{n \rightarrow \infty} T^{n} x_{0}=x^{*}$.

Proof. Assume $T^{n} x_{0} \neq T^{n+1} x_{0}$ for each $n \in N$. (If not, there is $n_{0} \in N$ such that $T^{n_{0}} x_{0}=T^{n_{0}+1} x_{0}$. This means that $x^{*}=T^{n_{0}} x_{0}$ is a fixed point of $T$ and $\left.\lim _{n \rightarrow \infty} T^{n} x_{0}=x^{*}\right)$.

Since $\left\{T^{n} x_{0}\right\}_{n=1}^{\infty}$ has an accumulation point $x^{*} \in X$, there exists a subsequence $\left\{T^{n_{i}} x_{0}\right\}$, $\lim _{n \rightarrow \infty} T^{n_{i}} x_{0}=x^{*}$.

$$
\left\{\mathcal{M}\left(T^{n} x_{0}, T^{n+1} x_{0}, T^{n+1} x_{0}, t\right)\right\} \quad \text { and } \quad\left\{\mathcal{N}\left(T^{n} x_{0}, T^{n+1} x_{0}, T^{n+1} x_{0}, t\right)\right\}
$$

are non-decreasing and non-increasing, respectively, and also bounded, thus

$$
\left\{\mathcal{M}\left(T^{n_{i}} x_{0}, T^{n_{i}+1} x_{0}, T^{n_{i}+1} x_{0}, t\right)\right\},\left\{\mathcal{M}\left(T^{n_{i}+1} x_{0}, T^{n_{i}+2} x_{0}, T^{n_{i}+2} x_{0}, t\right)\right\}
$$

and

$$
\left\{\mathcal{N}\left(T^{n_{i}} x_{0}, T^{n_{i}+1} x_{0}, T^{n_{i}+1} x_{0}, t\right)\right\},\left\{\mathcal{N}\left(T^{n_{i}+1} x_{0}, T^{n_{i}+2} x_{0}, T^{n_{i}+2} x_{0}, t\right)\right\}
$$

are convergent to a common limit, i.e.,

$$
\begin{aligned}
& \lim _{i \rightarrow \infty} \mathcal{M}\left(T^{n_{i}} x_{0}, T^{n_{i}+1} x_{0}, T^{n_{i}+1} x_{0}, t\right)=\lim _{i \rightarrow \infty} \mathcal{M}\left(T^{n_{i}+1} x_{0}, T^{n_{i}+2} x_{0}, T^{n_{i}+2} x_{0}, t\right) \\
& \lim _{i \rightarrow \infty} \mathcal{N}\left(T^{n_{i}} x_{0}, T^{n_{i}+1} x_{0}, T^{n_{i}+1} x_{0}, t\right)=\lim _{i \rightarrow \infty} \mathcal{N}\left(T^{n_{i}+1} x 0, T^{n_{i}+2} x_{0}, T^{n_{i}+2} x_{0}, t\right)
\end{aligned}
$$

for all $t>0$.

By the continuity of $T$, we have

$$
\lim _{i \rightarrow \infty} T^{n_{i}+1} x_{0}=\lim _{i \rightarrow \infty} T\left(T^{n_{i}} x_{0}\right)=T x^{*} .
$$

Suppose $x^{*} \neq T x^{*}$. Putting $y=T x, z=T x$ in (3.7.1) and (3.7.2), we have

$$
\mathcal{M}(x, T x, T x, .)<\mathcal{M}\left(x, T^{2} x, T^{2} x, .\right) \quad \text { and } \mathcal{N}(x, T x, T x, .)>\mathcal{N}\left(x, T^{2} x, T^{2} x, .\right)
$$

for every $x \neq T x$. So, by Lemma 3.3, there exists a continuous point $t_{0}$ of $\mathcal{M}\left(x^{*}, T x^{*}, T x^{*},.\right)$ and $\mathcal{N}\left(x^{*}, T x^{*}, T x^{*},.\right)$ such that

$$
\mathcal{M}\left(x^{*}, T^{2} x^{*}, T^{2} x^{*}, t_{0}\right)>\mathcal{M}\left(x^{*}, T x^{*}, T x^{*}, t_{0}\right) \text { and }
$$




$$
\mathcal{N}\left(x^{*}, T^{2} x^{*}, T^{2} x^{*}, t_{0}\right)<\mathcal{N}\left(x^{*}, T x^{*}, T x^{*}, t_{0}\right) .
$$

On the other hand, by Lemma 2.5 .

$$
\begin{aligned}
\mathcal{M}\left(x^{*}, T x^{*}, T x^{*}, t_{0}\right) & =\lim _{i \rightarrow \infty} \mathcal{M}\left(T^{n_{i}} x_{0}, T\left(T^{n_{i}} x_{0}\right), T\left(T^{n_{i}} x_{0}\right), t_{0}\right) \\
& =\lim _{i \rightarrow \infty} \mathcal{M}\left(T^{n_{i}+1} x_{0}, T^{n_{i}+2} x_{0}, T^{n_{i}+2} x_{0}, t_{0}\right) \\
& \geq \mathcal{M}\left(T x^{*}, T^{2} x^{*}, T^{2} x^{*}, t_{0}\right) \\
\mathcal{N}\left(x^{*}, T x^{*}, T x^{*}, t_{0}\right) & =\lim _{i \rightarrow \infty} \mathcal{N}\left(T^{n_{i}} x_{0}, T\left(T^{n_{i}} x_{0}\right), T\left(T^{n_{i}} x_{0}\right), t_{0}\right) \\
& =\lim _{i \rightarrow \infty} \mathcal{N}\left(T^{n_{i}+1} x_{0}, T^{n_{i}+2} x_{0}, T^{n_{i}+2} x_{0}, t_{0}\right) \\
& \leq \mathcal{N}\left(T x^{*}, T^{2} x^{*}, T^{2} x^{*}, t_{0}\right)
\end{aligned}
$$

are contradictions. Therefore, $x^{*}=T x^{*}$, i.e. $x^{*}$ is a fixed point of $T$.

Uniqueness follows from (3.7.1) and (3.7.2). Finally, we prove that $\lim _{n \rightarrow \infty} T^{n} x_{0}=x^{*}$.

Since $\lim _{i \rightarrow \infty} T^{n_{i}} x_{0}=x^{*}$ and $\lim _{i \rightarrow \infty} T^{n_{i}+1} x_{0}=T x^{*}=x^{*}$, by Lemma 2.5,

$$
\begin{aligned}
& \lim _{i \rightarrow \infty} \inf \mathcal{M}\left(T^{n_{i}} x_{0}, T^{n_{i}+1} x_{0}, T^{n_{i}+1} x_{0}, t\right) \geq \mathcal{M}\left(x^{*}, x^{*}, x^{*}, t\right)=1, \\
& \lim _{i \rightarrow \infty} \sup \mathcal{N}\left(T^{n_{i}} x_{0}, T^{n_{i}+1} x_{0}, T^{n_{i}+1} x_{0}, t\right) \leq \mathcal{N}\left(x^{*}, x^{*}, x^{*}, t\right)=0,
\end{aligned}
$$

for all $t>0$. So,

$$
\begin{aligned}
& \lim _{i \rightarrow \infty} \mathcal{M}\left(T^{n_{i}} x_{0}, T^{n_{i}+1} x_{0}, T^{n_{i}+1} x_{0}, t\right)=1, \\
& \lim _{i \rightarrow \infty} \mathcal{N}\left(T^{n_{i}} x_{0}, T^{n_{i}+1} x_{0}, T^{n_{i}+1} x_{0}, t\right)=0,
\end{aligned}
$$

for all $t>0$. For any $n \in N$ with $n>n_{1}$, there exists $n_{i}$ with $n_{i+1} \geq n>n_{i}$.

By (3.7.1) and (3.7.2), we have

$$
\begin{aligned}
& \mathcal{M}\left(T^{n} x_{0}, x^{*}, x^{*}, t\right) \geq \min \left\{\mathcal{M}\left(T^{n-1} x_{0}, T^{n} x_{0}, T^{n} x_{0}, t\right), 1, \mathcal{M}\left(T^{n} x_{0}, x^{*}, x^{*}, t\right)\right\} \\
& \geq \min \left\{\mathcal{M}\left(T^{n-1} x_{0}, T^{n} x_{0}, T^{n} x_{0}, t\right), \mathcal{M}\left(T^{n-2} x_{0}, T^{n-1} x_{0}, T^{n-1} x_{0}, t\right), M\left(T^{n-2} x_{0}, x^{*}, x^{*}, t\right)\right\} \\
& \geq \min \left\{\mathcal{M}\left(T^{n-2} x_{0}, T^{n-1} x_{0}, T^{n-1} x_{0}, t\right), \mathcal{M}\left(T^{n-2} x_{0}, x^{*}, x^{*}, t\right)\right\} \\
& \geq \cdots \geq \min \left\{\mathcal{M}\left(T^{n_{i}} x_{0}, T^{n_{i}+1} x_{0}, T^{n_{i}+1} x_{0}, t\right), \mathcal{M}\left(T^{n_{i}} x_{0}, x^{*}, x^{*}, t\right)\right\} \\
& \mathcal{N}\left(T^{n} x_{0}, x^{*}, x^{*}, t\right) \leq \max \left\{\mathcal{N}\left(T^{n-1} x_{0}, T^{n} x_{0}, T^{n} x_{0}, t\right), 0, \mathcal{N}\left(T^{n} x_{0}, x^{*}, x^{*}, t\right)\right\} \\
& \leq \max \left\{\mathcal{N}\left(T^{n-1} x_{0}, T^{n} x_{0}, T^{n} x_{0}, t\right), \mathcal{N}\left(T^{n-2} x_{0}, T^{n-1} x_{0}, T^{n-1} x_{0}, t\right), \mathcal{N}\left(T^{n-2} x_{0}, x^{*}, x^{*}, t\right)\right\} \\
& \leq \max \left\{\mathcal{N}\left(T^{n-2} x_{0}, T^{n-1} x_{0}, T^{n-1} x_{0}, t\right), \mathcal{N}\left(T^{n-2} x_{0}, x^{*}, x^{*}, t\right)\right\} \\
& \leq \cdots \leq \max \left\{\mathcal{N}\left(T^{n_{i}} x_{0}, T^{n_{i}+1} x_{0}, T^{n_{i}+1} x_{0}, t\right), \mathcal{N}\left(T^{n_{i}} x_{0}, x^{*}, x^{*}, t\right)\right\}
\end{aligned}
$$

Letting $n \rightarrow \infty\left(n_{i} \rightarrow \infty\right)$, we have

$$
\lim _{n \rightarrow \infty} \mathcal{M}\left(T^{n} x_{0}, x^{*}, x^{*}, t\right) \geq 1 \text { and } \lim _{n \rightarrow \infty} \mathcal{N}\left(T^{n} x_{0}, x^{*}, x^{*}, t\right) \leq 0
$$

for all $t>0$.

Hence, $\lim _{n \rightarrow \infty} T^{n} x_{0}=x^{*}$. 


\section{References}

[1] Atanassov, K. (1983). Intuitionistic fuzzy sets, VII ITKR's session, Sofia, June 1983 (Deposed in central Science-Technical Library of Bulg. Academy of Science, 1697/84) (in Bulgarian). Reprinted: Int. J. Bioautomation, 2016, 20(S1), S1-S6 (in English).

[2] Banach, S. (1932). Theorie des Operations Lineaires, Warsaw: Monografj Mathematyczne.

[3] Edelstein, M. (1962). On fixed and periodic points under contractive mapping. J London Math Soc., 37, 74-79.

[4] George, A., \& Veeramani, P. (1994). On some results in fuzzy metric spaces, Fuzzy Sets and Systems, 64, 395-399.

[5] Gregori, V., \& Sapena, A. (2002). On fixed point theorems in fuzzy metric spaces, Fuzzy Sets and Systems, 125, 245-252.

[6] Kramosil, I., \& Michalek, J. (1975). Fuzzy metric and statistical metric spaces, Kybernotice, 11, 326-334.

[7] Park, J. H. (2004). Intuitionistic fuzzy metric spaces, Chaos, Solitons Fractals, 22, 1039-1046.

[8] Sedghi, S., \& Shobe, N. (2006). Fixed point theorem in $\mathcal{M}$-fuzzy metric spaces with property (E), Advances in Fuzzy Mathematics, 1(1), 55-65.

[9] Zadeh, L. A. (1965). Fuzzy sets, Information and Control, 8, 338-353. 